\title{
Frequency mixing in nonlinear interaction of one-way edge-modes of topological photonic crystals
}

Lan, Zhihao, You, Jian Wei, Panoiu, Nicolae

Zhihao Lan, Jian Wei You, Nicolae C. Panoiu, "Frequency mixing in nonlinear interaction of one-way edge-modes of topological photonic crystals," Proc. SPIE 11201, SPIE Micro + Nano Materials, Devices, and Applications 2019, 1120108 (31 December 2019); doi: 10.1117/12.2539874

SPIE. Event: ANZCOP, 2019, Melbourne, Australia 


\title{
Frequency mixing in nonlinear interaction of one-way edge-modes of topological photonic crystals
}

\author{
Zhihao Lan, Jian Wei You, and Nicolae C. Panoiu \\ Department of Electronic and Electrical Engineering, University College London, Torrington Place, London WC1E 7JE, United Kingdom \\ Authore-mail address: z.lan@ucl.ac.uk, j.you@ucl.ac.uk, and n.panoiu@ucl.ac.uk.
}

\begin{abstract}
We investigate topological photonic crystals specially designed such that the frequency band gaps appear around $\omega_{0}, 2 \omega_{0}, 3 \omega_{0}$ and, more importantly, each band gap contains exactly one unidirectional edge mode. These one-way edge modes are then utilized to implement key nonlinear frequency mixing processes, such as second- and third-harmonic generation.
\end{abstract}

\section{Introduction}

Topological photonics [1] has recently attracted great interest due to the opportunity it provides for robust light propagation and manipulation. A well-known feature of optics is the existence of nonlinearity in many optical materials, which is expected to greatly expand the horizon of topological photonic systems. Indeed, lattice edge solitons [2], nonlinear control [3] and mapping [4] of photonic topological edge states, and the potential to enhance harmonic generation [5] have been demonstrated. Despite these advances, the feasibility of achieving nonlinear optical mixing via the edge states of topological photonic crystals (PhCs) has not been explored yet. Here we show that such nonlinear frequency mixing processes, e.g., second- and third-harmonic generation (SHG and THG) are indeed possible by properly designing the topological properties of the frequency band gaps.

\section{Results and Discussion}

\subsection{The setup and the emergence of one-way edge mode}

We consider two-dimensional (2D) PhCs with hexagonal symmetry whose unit cell contains one cylinder of nonlinear material embedded in a magnetic background material (see Fig. 1a). The permeability tensor of the magnetic material under an external magnetic field possesses off-diagonal components (i.e., $\mu_{\text {off-diag }} \neq 0$ ). Due to the broken time-reversal symmetry, the frequency bands could acquire nonzero Chern number. We show in Fig. 1c one example where certain gaps appear around $\omega=0.2,0.4$, and 0.6 and more importantly, all the three gaps possess nontrivial gap Chern number of $C_{\mathrm{g}}=1$. According to the bulk-edge correspondence, one-way edge states will emerge within the frequency gaps for systems with finite size. We show in Fig. 1d the photonic band structure of a PhC strip with 30 unit cells along the $y$-axis and periodic along the $x$-axis. As expected, one-way edge states indeed emerge. Fig. 1e highlights the key feature of the edge state - exponential decay of the field away from the edge.
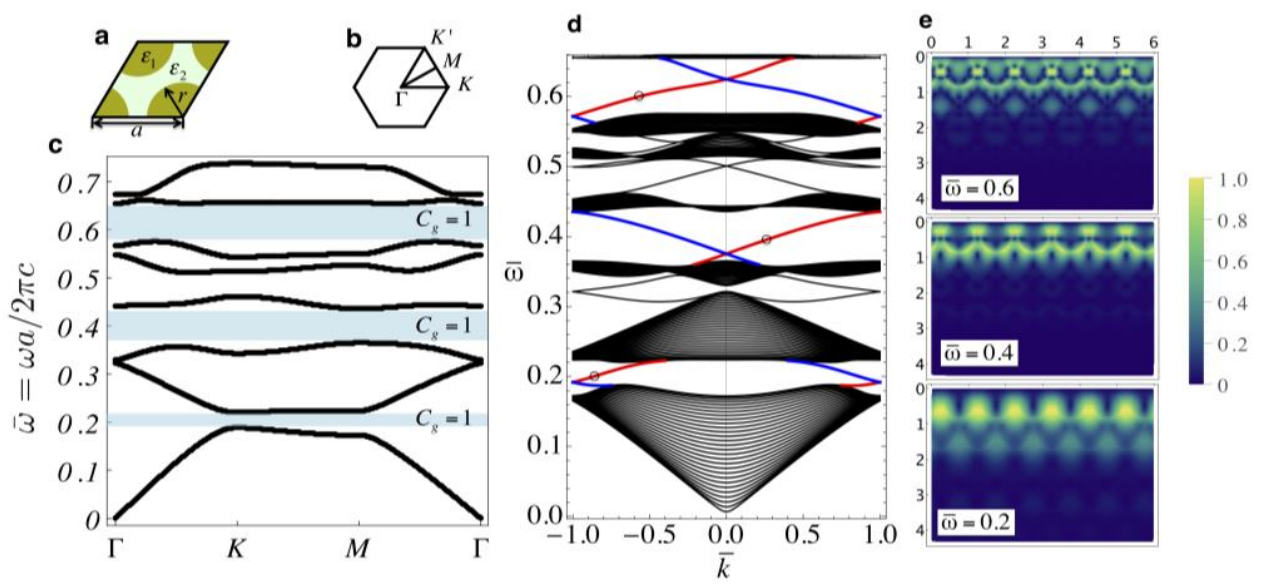

Fig. 1. (a) The unit cell and (b) the first Brillouin zone of the PhC. (c) Topological frequency band gaps with gap Chern number of $C_{\mathrm{g}}=1$ at $\varepsilon_{1}=3, \varepsilon_{2}=20, r=0.42 \mathrm{a}$ and $\mu_{\mathrm{off}-\mathrm{diag}}=0.8$. (d) The emergence of one-way edge modes for a finite size system, where red and blue modes are formed on the top and bottom boundaries of the PhC. (e) Field profiles of the three edge modes marked by circles in (d). 


\subsection{SHG and THG upon edge-mode interaction}

The one-way edge modes as shown in Fig. 1d can be used to implement the nonlinear frequency mixing processes, such as SHG and THG. We show in Fig. 2a the simulated field profile intensities of $E_{1}, E_{2}$, and $E_{3}$ at $\varpi=0.2,0.4$, and 0.6 , where $E_{1}$ is induced by an external source (red star) whereas $E_{2}$ and $E_{3}$ are generated by the corresponding nonlinear polarizations. One can observe that the field profiles of $E_{1}, E_{2}$, and $E_{3}$ are indeed the same as those of the edge mode profiles shown in Fig. 1e, indicating that the edge modes are indeed nonlinearly interacting via the SHG and THG. To further validate this statement, we study the phase matching effects on the SHG and THG by scanning the excitation frequency of the fundamental mode. Due to the edge mode dispersions (see left panels of Figs. 2b, 2c), a wavevector mismatch $\Delta k$ of the nonlinear process will cause spatial oscillations of the generated harmonic fields (see Fig. 2a). The oscillation period is given by $\Lambda=2 \pi / \Delta k$. We present the numerically extracted oscillation periods and the theoretical predictions from the edge mode dispersions in the right panels of Fig. 2b, 2c. The excellent agreement confirms SHG and THG purely via nonlinear interaction of edge modes indeed occur in our setup.

a

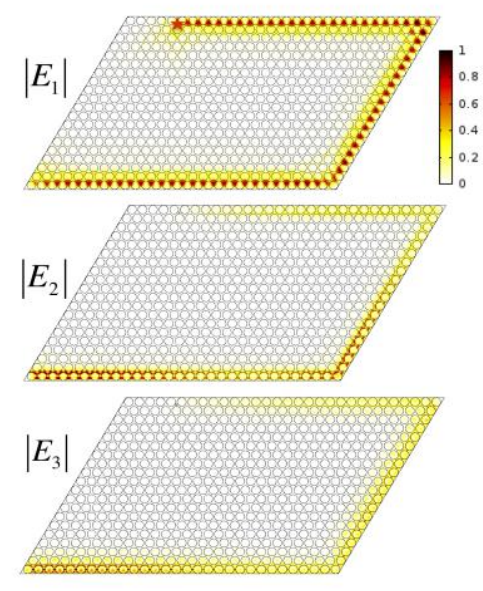

b
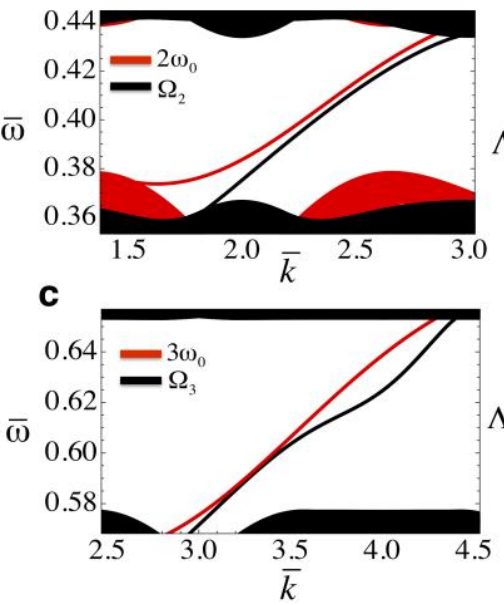

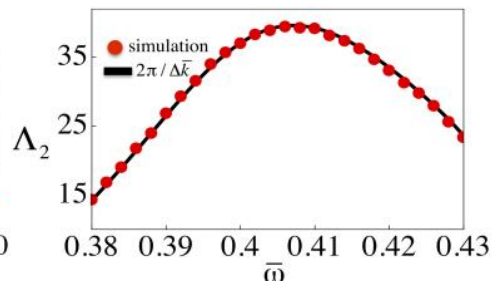

$\bar{\omega}$

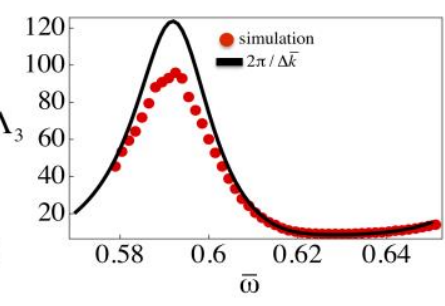

Fig. 2. (a) Simulated field profiles of SHG and THG via interactions of topological edge modes. (b) Left panel shows the edge mode dispersions of the fundamental $\left(\omega_{0}\right)$ and harmonic $\left(\Omega_{2}=2 \omega_{0}\right)$ waves. The right panel shows the comparison between the theoretically calculated $\left(\Lambda_{2}=2 \pi / \Delta k\right)$ and numerically extracted oscillation period of $\mathrm{E}_{2}$. (c) Same as (b) but for THG. Note the discrepancy between the numerical and theoretical results around the peak is due to inherent limitations of numerical simulations at very small $\Delta k$.

\section{Conclusions}

We have presented proof-of-principle demonstration that topology-protected nonlinear frequency mixing processes via the edge states of topological PhCs, such as SHG and THG, can be achieved. Further phenomena, such as slowlight enhancement of nonlinear interactions and harmonic generation upon interaction of backward-propagating (left-handed) edge modes can also be realized within the setup we presented here [6]. The concept of topologyprotected nonlinear frequency mixing processes can also be applied to other fields such as plasmonics and acoustics.

\section{References}

[1] T. Ozawa, H. M. Price, A. Amo, N. Goldman, M. Hafezi, L. Lu, M. Rechtsman, D. Schuster, J. Simon, O. Zilberberg, and I. Carusotto, "Topological photonics," Rev. Mod. Phys. 91, 015006 (2019).

[2] Y. Lumer, Y. Plotnik, M. C. Rechtsman, and M. Segev. "Self- Localized States in Photonic Topological Insulators," Phys. Rev. Lett. 111, 243905 (2013).

[3] D. A. Dobrykh, A. V. Yulin, A. P. Slobozhanyuk, A. N. Poddubny, and Y. S. Kivshar, "Nonlinear Control of Electromagnetic Topological Edge States," Phys. Rev. Lett. 121, 163901 (2018).

[4] D. Smirnova, S. Kruk, D. Leykam, E. Melik-Gaykazyan, D. -Y. Choi, and Y. Kivshar, "Nonlinear mapping of photonic topological edge states," arXiv:1811.12130.

[5] Y. Wang, L.-J. Lang, C. H. Lee, B. Zhang, and Y. D. Chong, "Topologically enhanced harmonic generation in a nonlinear transmission line metamaterial," Nat. Commun. 10, 1102 (2019).

[6] Z. Lan, J. W. You, and N. C. Panoiu, "Nonlinear one-way edge-mode interactions for frequency mixing in topological photonic crystals," (to be submitted). 